
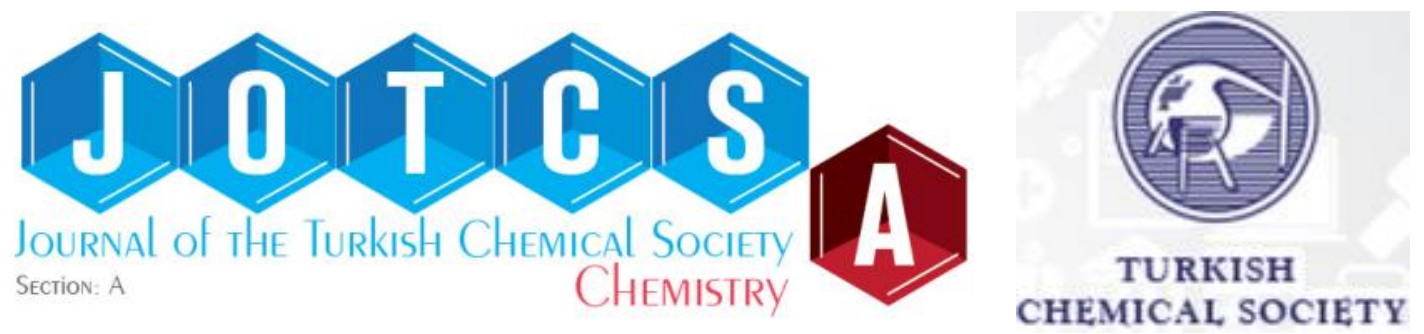

\title{
Molecular Interaction Between Cationic Polymer Polyethyleneimine and Rose Bengal Dye: A Spectroscopic Study
}

\author{
Tuğba BAYRAKTUTAN ${ }^{*} \otimes$ iD
}

${ }^{1}$ Department of Biochemistry, Faculty of Arts and Science, Iğdır University, Iğdır, Turkey

\begin{abstract}
The binding mechanism and polymer-fluorescence probe interactions between polyethyleneimine (PEI) and Rose Bengal (RB) were investigated by using UV-Vis absorption, steady-state and time-resolved fluorescence spectroscopy techniques. The spectroscopic data indicated that unusual interactions and binding constant was calculated at $3.75 \times 10^{2} \mathrm{M}^{-1}$ with high linearity for the PEI-RB system. The photophysical parameters of the dye, such as band shifts, fluorescence quantum yields, and fluorescence lifetimes, were determined. Non-radiative $\left(k_{n r}\right)$ and radiative $\left(k_{r}\right)$ rate constants were calculated and then compared.
\end{abstract}

Keywords: Polyethyleneimine, Rose Bengal, Absorption and Fluorescence Spectroscopy, Dye-Polymer Interaction

Submitted: December 28, 2018. Accepted: July 10, 2019.

Cite this: Bayraktutan T. Molecular Interaction Between Cationic Polymer Polyethyleneimine and Rose Bengal Dye: A Spectroscopic Study. JOTCSA. 2019;6(3):311-8.

DOI: https://doi.org/10.18596/jotcsa.504528.

*Corresponding author: E-mail: tugba.bayraktutan@igdir.edu.tr.

\section{INTRODUCTION}

Among the studies on macromolecule-small molecule interactions, comprehensive studies are available on dye compounds and binding through water-soluble synthetic or natural polymers. Many dyes were found to exhibit very different binding properties to unmodified PEI. Previous studies in this area have shown that the binding affinity of polyethyleneimine (PEI) is suitable ( 1 , 2). Among the cationic polymers, polyethyleneimine (PEI) is an applicable transfection reactant derived from the polyamine group (3). It is a polymer with linear or branched forms. PEI acts as a good polymer chelating agent due to its water solubility and suitable molecular weights. It is a polymer where chelating properties are examined by using potentiometric and spectrophotometric methods $(4,5)$. In this study, a branched PEI containing primary, secondary, and tertiary amino groups at approximately $1 / 4,1 / 2$ and $1 / 4$ rates respectively, were used (6). In this study, PEI provides a change in the spectral properties for Rose Bengal (RB) dye through $\Pi-\Pi$ stacking and electrostatic interaction.
Rose Bengal (RB) is an anionic dye derived from xanthene. It is a water-soluble, xanthene derived, an anionic dye, which has a high absorbance coefficient in the UV-visible region and high sensitivity to light. The tendency to transfer long life radicals from the triplet excited state and intersystem crossing efficiency is high $(7,8)$. Since it is a dye that is highly sensitive to a micro-environment, it is much preferred in photophysical studies.

This study aims to examine the polymer-dye interactions between Polyethylenimine (PEI) and Rose Bengal (RB) by using UV-Vis absorption and fluorescence spectroscopy techniques. In this context, PEI is a good candidate because polyamine polymers have a high concentration of amine groups, which provides an effective macromolecular environment in the aqueous solution. In this study, we will provide RB-PEI interaction, and the photophysical parameters will be discussed and an attempt presented for the polymer-dye interaction mechanism. The formation of complex and aggregation in the dyepolymer interactions is one of the most common 
situations in the literature. The dye-polymer studies are a powerful tool for determining the binding region, the driving forces in the binding, conformational changes of the polymer and which polymer has an affinity to the dye. In this respect, this study is significant in terms of adding a dyepolymer relationship to the literature.

\section{EXPERIMENTAL}

\section{Chemicals}

Rose Bengal and Polyethyleneimine (the molecular structures are shown in the Figure $1 \mathrm{a}$ and $1 b$, respectively) were purchased from Sigma. Methanol was purchased from Fluka. RB and PEI were stored in the dark as concentrated stock solutions of $1.0 \mathrm{mM}$ in methanol. The chemicals were used without further purification. All experiments were done in distilled water.<smiles>NCCNCCN(CCN)CCN(CCNCCN)CCN(CCNCCN)CCNCCN</smiles>

Figure 1. Molecular structures of (a) Polyethyleneimine and (b) Rose Bengal (Rose Bengal b sodium salt).

\section{Equipments}

Absorption and fluorescence spectra of samples were taken with Lambda 35 UV / Vis Spectrophotometer and Shimadzu RF-5301PC, respectively. Time-resolved fluorescence measurements were recorded in the Photon Technology Instrument (PTI) Time Master TM3. The excitation and emission slits were adjusted at $1.0 \mathrm{~nm}$. The absorption spectra were recorded in the region of $455 \mathrm{~nm}$ to $620 \mathrm{~nm}$ at room temperature. For the fluorescence spectra, the system was excited at $520 \mathrm{~nm}$ and the fluorescence spectrum was recorded between 525 $\mathrm{nm}$ and $620 \mathrm{~nm}$. A more detailed description of the method time depending fluorescence measurements is given other where (9).

\section{Fluorescence Quantum Yield Measurements} Fluorescence quantum yields $\left(\Phi_{f}\right)$ were calculated using a reference solution and using equation 1 .

$$
\phi_{S}=\phi_{r}\left(\frac{D_{S}}{D_{r}}\right)\left(\frac{n_{S}}{n_{r}}\right)^{2}\left(\frac{1-10^{o D r}}{1-10^{o D s}}\right)
$$

In equation $1, D_{s}$ and $D_{r}$ refer to the integrated area under the corrected fluorescence spectra for sample and reference, $n_{s}$ and $n_{r}$ are refractive indexes of sample and reference solutions, respectively. The ODs are the sample and the ODr is the reference optical density read at the excitation wavelength $(10,11)$. The fluorescence quantum yield of 1 uM Rh101 is 1.00 in methanol (12).

\section{RESULT AND DISCUSSION}

\section{Effects of PEI on the absorption spectra of RB}

PEI does not show any absorbance in the visible region. The UV-Vis absorption spectra of the $R B$ (2.0 uM) at various PEI concentrations changing from 0.02 to $0.90 \mathrm{mg} / \mathrm{mL}$ are shown in Figure 2 . Figures $2 \mathrm{a}$ and $2 \mathrm{~b}$ show the absorption spectra and normalized absorption spectra of the dye at room temperature and in a distilled water medium, respectively. RB shows maximum fluorescence absorption at $549 \mathrm{~nm}$. When the various concentration polymer solutions are titrated to the dye solution, spectral shifts occur as a function of the PEI concentration. The biggest shift of about $15 \mathrm{~nm}$ was observed at the highest PEI concentration. 

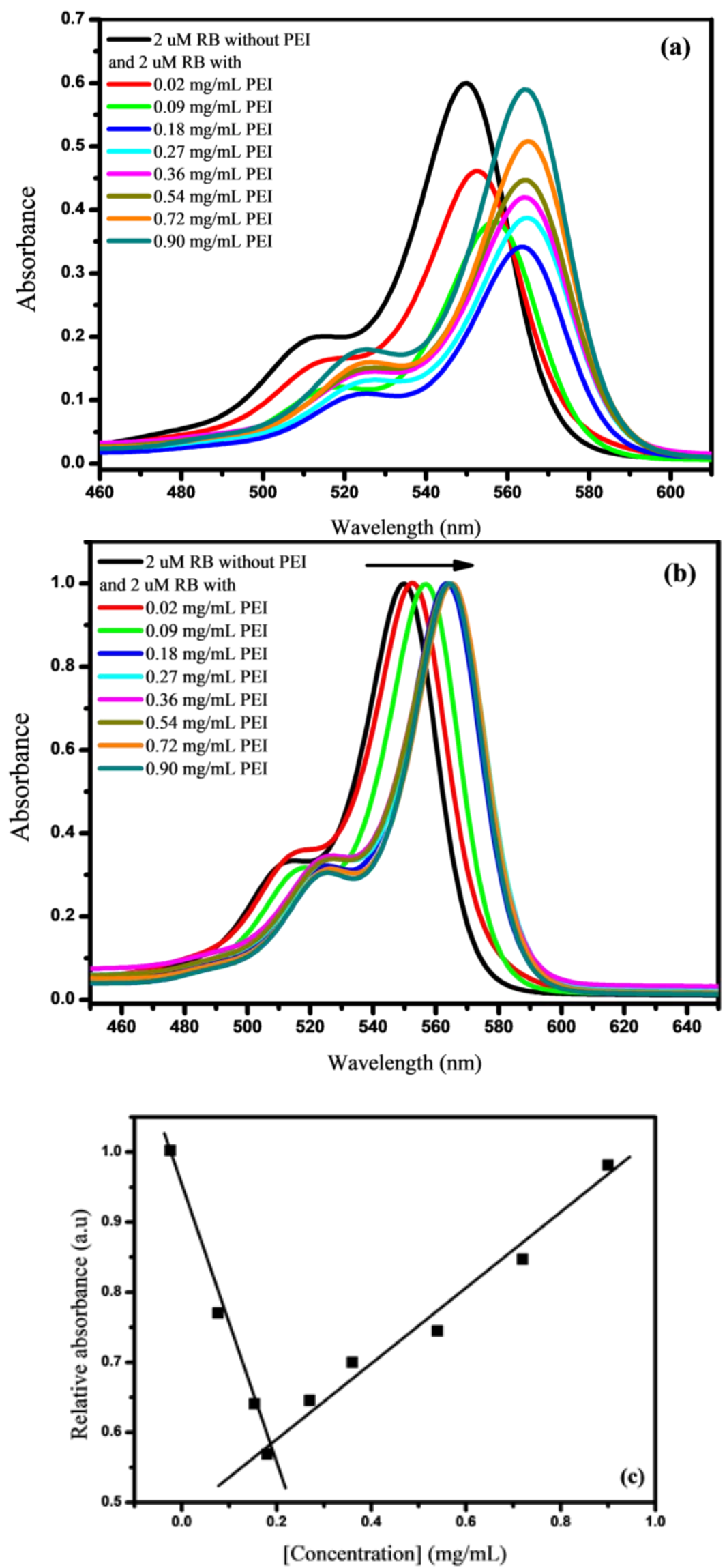

Figure 2. (a) Observed and (b) normalized UV-Vis absorption spectra of RB with various concentrations of PEI in distilled water. (c) Relation between the relative absorbance and the ratio of as a function of concentration of PEI. 
According to the theory of molecular exciton, the transition moments are final for the electric dipole changeover from the ground state to the excited state or from the ground state to the higher energized excited state. It is known that a linear transition dipole will cause dimer, aggregation, or higher complexity. Such interactions always result in strong red shifts for the dimer, aggregate, and complex transition $(13,14)$. The absorption band of RB-PEI expands about $5 \mathrm{~nm}$, and a strong red shift is observed in the comparative absorption bands of the RB alone. These spectral changes confirm the RB attaches to the polymer chain of PEI (15). These interactions lead to a decrease in absorbance at lower PEI concentrations and an increase in absorbance in higher PEI concentrations. This is clearly illustrated in the graph in Figure 1c showing the relative absorbance values based on the PEI concentrations. The change of the interaction of the resulting complex is clearly shown.

Determination of binding constant and evaluation of the quantities of binding. Absorbance measurements were made for pure dye and PEI-dye mixture. For dye, Adye refers to absorbance of RB dye alone; $A$ refers to absorbance for the PEI- RB and the difference occured in absorbance is calculated with the following formula:

$A-A_{\text {dye }}=\Delta A=C_{B}\left(\varepsilon_{\text {complex }}-\varepsilon_{\text {dye }}\right)=C_{B} \Delta \varepsilon($ Eq. 2$)$

where $C_{B}$ is the concentration of bound dye and $\Delta \varepsilon$ is the change of the molar absorption coefficient of the bound dye or complex and the free dye. The balance of complex formation between polymer and dye is shown as follows

$$
\text { PEI }+ \text { dye } \stackrel{K}{\leftrightarrow} \text { Complex }
$$

where $K$ is the binding constant. If the complex is considered to be composed of 1 : 1

$$
K=\frac{[\text { complex }]}{C_{F}[\text { PEI }]_{\text {free }}}=\frac{C_{B}}{C_{F}[P E I]_{\text {free }}}
$$

where $C_{F}$ refer to the free dye concentration. When the FEI is overdone, $[P E I]_{\text {free }} \sim[P E I]_{\text {total }}$ and represents $[P E I]_{\text {total }}$ as $[P E I]$ be simplified;

$$
K=\frac{C_{B}}{C_{F}[P E I]} \text { or } \frac{C_{B}}{C_{F}}=K[P E I]
$$

The total dye concentration $C_{T}$ is shown by

$$
C_{T}=C_{B}+C_{F}
$$

By dividing Eq. 5 by Eq.2, we get the following:

$$
\frac{C_{T}}{\Delta A}=\frac{C_{B}+C_{F}}{C_{B} \Delta \varepsilon}
$$

By simplifying Eq. 6 with the replacement of the value of $C_{B} / C_{F}$ from Eq.4 we have

$$
\frac{C_{T}}{\Delta A}=\frac{1}{\Delta \varepsilon}\left(1+\frac{1}{K[P E I]}\right.
$$

Eq. 7 is the linking of experimentally known or quantifiable amounts of $\Delta A$ to $K$ and $\Delta \varepsilon$ to the binding study (16). Figure 3 shown the plot of Eq.7 (CT/ $\triangle \mathrm{A}$ vs $1+1 / \mathrm{K}[\mathrm{PEI}])$ for system. The experimental data were given in Table 1.

Table 1. Binding Constant and Extinction Coefficients for the PEI-Rose Bengal System

\begin{tabular}{|c|c|c|c|c|}
\hline SYSTEM & $\begin{array}{c}\boldsymbol{\varepsilon}_{\text {dye }} \times \mathbf{1 0}^{5} \\
\left(\mathbf{M}^{-1} \mathbf{c m}^{-1}\right)\end{array}$ & $\begin{array}{c}\Delta \varepsilon \times \mathbf{1 0}^{5} \\
\left(\mathbf{M}^{-1} \mathbf{c m}^{-1}\right)\end{array}$ & $\begin{array}{c}\varepsilon_{\text {complex }} \mathbf{X 1 0 ^ { 5 }} \\
\left(\mathbf{M}^{-1} \mathbf{c m}^{-1}\right)\end{array}$ & $\begin{array}{c}\mathbf{K} \times \mathbf{1 0}^{\mathbf{2}} \\
\left(\mathbf{M}^{-1}\right)\end{array}$ \\
\hline PEI-RB & 3.00 & 1.81 & 1.19 & 3.75 \\
\hline
\end{tabular}

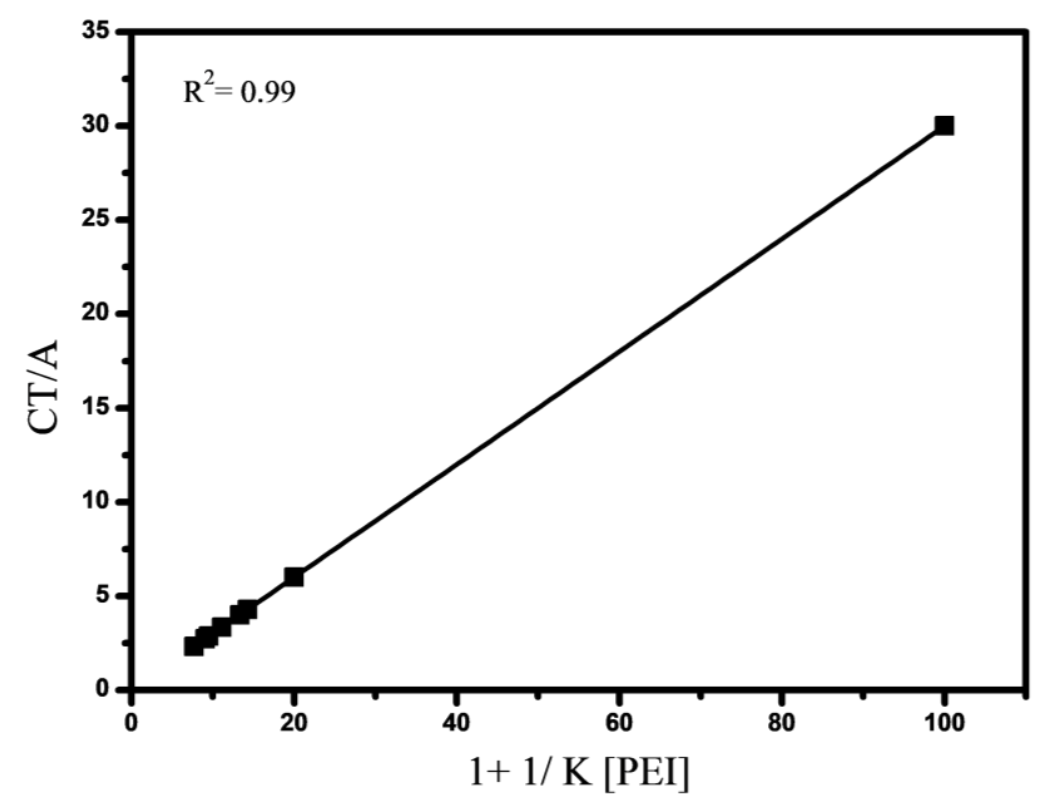

Figure 3. Plot of Eq. (6) for RB-PEI system. 


\section{Effects of PEI on the fluorescence spectra of RB}

The fluorescence spectra of the RB (2.0 uM) in different PEI concentrations ranging from 0.02 to $0.90 \mathrm{mg} / \mathrm{mL}$ are shown in Figure 4. Figures $4 \mathrm{a}$ and $b$ show the fluorescence spectra and normalized fluorescence spectra of the dye in a distilled water medium and at room temperature, respectively. PEI does not release any emissions during the absorbance. The fluorescence band of the RB in distilled water was monitored at 560 $\mathrm{nm}$. This band was initially quenched by forming its complex structures depending on the PEI concentration. As the concentration of the PEI continued to increase, fluorescence began to increase. This is clearly shown in Figure $4 \mathrm{c}$ in the graph of relative fluorescence values to the PEI concentration. The increased fluorescence of complex is an indication that the existing interaction converts the RB molecules to aggregates $(17,18)$. It is known that the formation of aggregate blocks non-radiative decay and increases the fluorescence of dye by intramolecular rotation in the phenyl rings (19, 20). In view of the emission spectra of the RB, the enhanced fluorescence resulting from the increase in the presence of PEI was proven to interact with the tendency of dye. The bands at longer wavelengths arise from the excimer emission of the interplayed aromatic units and aggregate $(21,22)$.
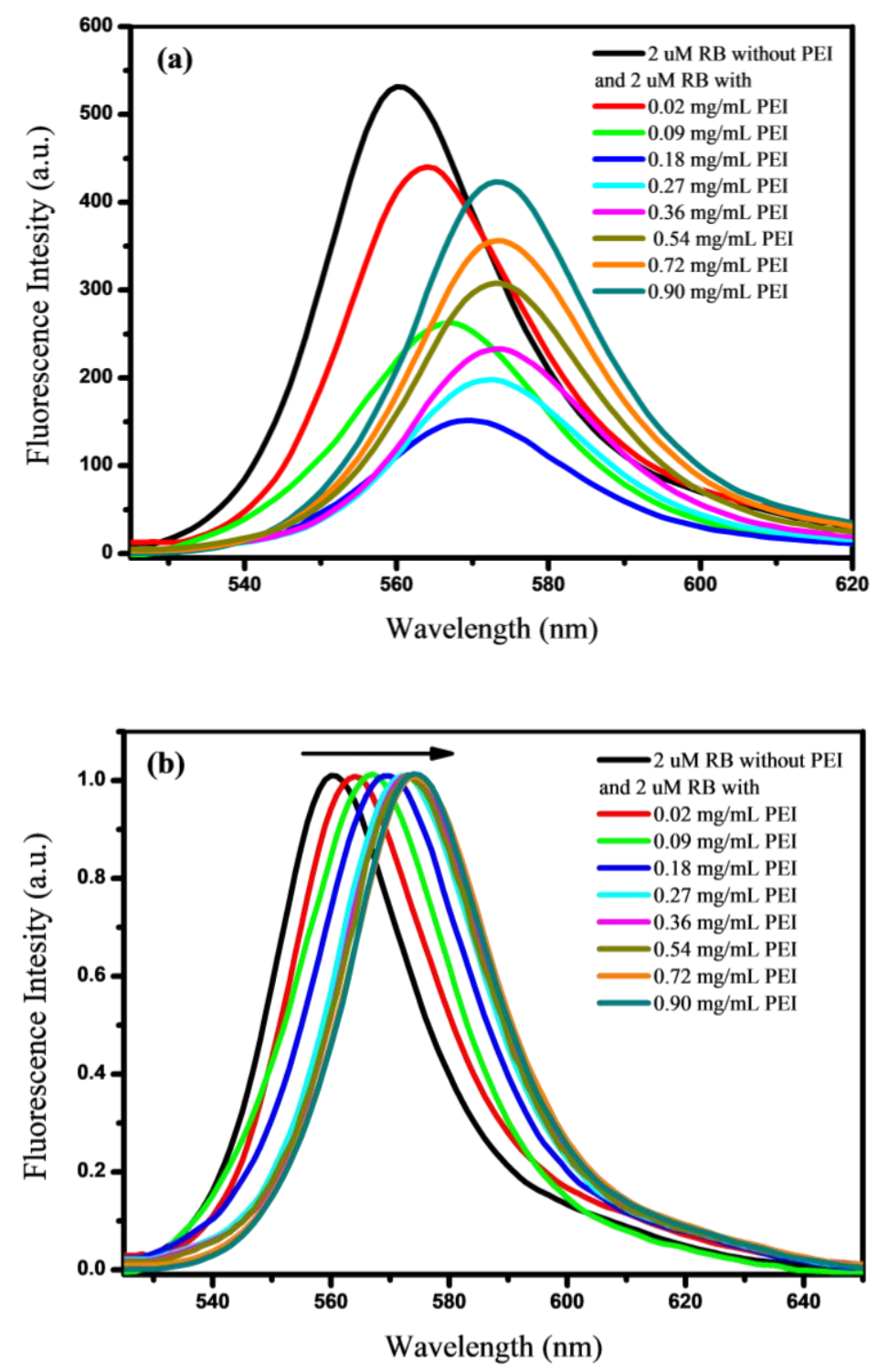


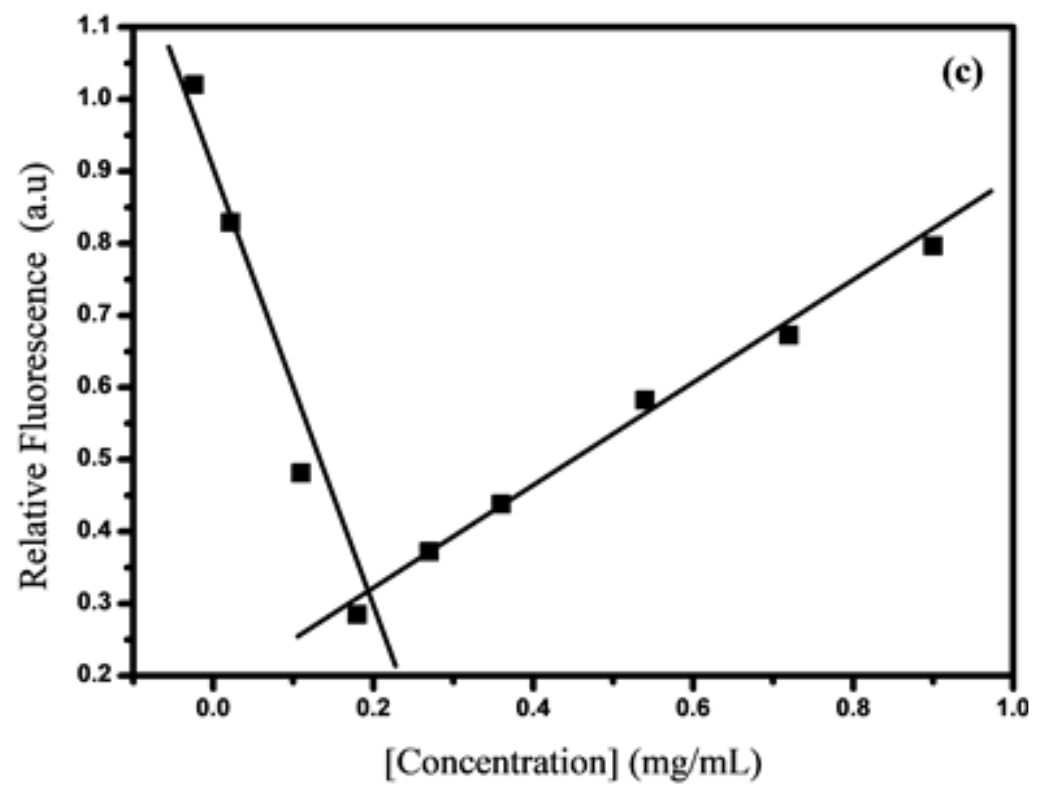

Figure 4. (a) Observed and (b) normalized fluorescence spectra of the RB with various concentrations of PEI in distilled water. (c) Relation between the relative fluorescence and the ratio of as a function of concentration of PEI.

\section{Determination of photophysical parameters for PEI-RB system}

The dye-polymer interaction resulted in the band shifting absorption. Similarly, the fluorescence spectra altered the fluorescence intensity of the $\mathrm{RB}$ and changed the fluorescence quantum yields $\left(\Phi_{f}\right)$ of the dye (Table 2 ). The data from Table 2 indicates that the quantum yield of RB decreases or increases depending on the fluorescence. Meanwhile, the fluorescence quantum efficiency varies greatly by the nature of the adhesion (23). The fluorescence lifetimes of the dye increased with the concentration of the polymer. The dye molecules are more stable when they interact with the polymer and this increases the life time values due to the molecule spending more time on excited energy levels (24). The emission band maximum of the dye, which shifted from 560 to $574 \mathrm{~nm}$, proves the formation of the $n$-stacked binding complex (25). The fluorescence lifetime of the dye is calculated and shown in Table 2. Fluorescence lifetime and fluorescence quantum yield are associated with the non-radiative $\left(k_{n r}\right)$ and radiative $\left(\mathrm{k}_{\mathrm{r}}\right)$ rate constants given in Equations 8 and $9(26,27)$.

Table 2. Photophysical parameters of RB in distilled water and PEI aqueous solutions.

\begin{tabular}{|c|c|c|c|c|c|c|c|c|}
\hline $\begin{array}{c}\text { [PEI] } \\
(\mathrm{mg} / \mathrm{mL})\end{array}$ & $\begin{array}{c}\lambda_{\text {Abs }} \\
(\mathbf{n m})\end{array}$ & $\begin{array}{c}\lambda_{\mathrm{F}} \\
(\mathbf{n m})\end{array}$ & $\begin{array}{c}\phi_{\mathrm{f}} \\
\left(\times 10^{-3}\right)\end{array}$ & $\begin{array}{c}T_{\mathbf{f}} \\
\text { (ns) }\end{array}$ & $x^{2}$ & $\begin{array}{c}k_{r}\left(s^{-1}\right) \\
\times 10^{9}\end{array}$ & $\begin{array}{c}\mathbf{k}_{\mathrm{nr}}\left(\mathrm{s}^{-1}\right) \\
\times 10^{9}\end{array}$ & $\mathbf{k}_{\mathbf{n r}} / \mathbf{k}_{\mathrm{r}}$ \\
\hline $\begin{array}{l}\text { Distilled } \\
\text { water }\end{array}$ & 550 & 560 & 18.0 & 0.095 & 1.2 & 18.95 & 10.34 & 54.5 \\
\hline 0.02 & 552 & 563 & 19.0 & 0.123 & 1.1 & 15.45 & 7.975 & 51.6 \\
\hline 0.09 & 557 & 566 & 17.0 & 0.185 & 1.0 & 9.19 & 5.313 & 57.8 \\
\hline 0.18 & 563 & 569 & 12.0 & 0.222 & 1.0 & 5.40 & 4.450 & 82.3 \\
\hline 0.27 & 564 & 571 & 16.0 & 0.246 & 1.0 & 6.50 & 4.000 & 61.5 \\
\hline 0.36 & 564 & 573 & 18.0 & 0.338 & 1.1 & 5.32 & 2.905 & 54.6 \\
\hline 0.54 & 565 & 573 & 20.0 & 0.371 & 1.2 & 5.39 & 2.641 & 49.0 \\
\hline 0.72 & 565 & 573 & 22.0 & 0.413 & 1.2 & 5.33 & 2.368 & 44.4 \\
\hline 0.90 & 566 & 574 & 23.0 & 0.455 & 1.1 & 5.05 & 2.147 & 42.5 \\
\hline
\end{tabular}

$$
\begin{array}{cc}
\mathrm{K}_{\mathrm{r}}=\Phi_{\mathrm{f}} / \mathrm{T}_{\mathrm{f}} & (\text { Eq.8) } \\
\mathrm{K}_{\mathrm{nr}}=1 / \mathrm{T}_{\mathrm{f}}-\mathrm{K}_{\mathrm{r}} \quad \text { (Eq.9) }
\end{array}
$$

Using these equations, $\mathrm{k}_{\mathrm{nr}} / \mathrm{k}_{\mathrm{r}}$ values were calculated for dye in the PEI solutions and distilled water. Table 2 shows that the $\mathrm{knr}_{\mathrm{n}} / \mathrm{k}_{\mathrm{r}}$ values for dye were higher at PEI concentrations of 0.18 $\mathrm{mg} / \mathrm{mL}$. On the other hand, $\mathrm{k}_{\mathrm{nr}} / \mathrm{k}_{\mathrm{r}}$ values are often above the average in similar systems. In addition, high $\mathrm{k}_{n r} / \mathrm{k}_{\mathrm{r}}$ values of RB show that the main excited-state deactivation pathway is internal conversion (28).

\section{CONCLUSION}

In the present study, the effects of PEI on the photophysical properties of RB dye were examined using spectroscopic techniques. The interactions of the RB dye and PEI in distilled water caused differences in the photophysical and spectroscopic properties of the dyes. RB (2 uM) in 
the presence of PEI displays strong red shifts and changes of absorption and emission band intensities when compared to that in distilled water, which are assigned to the dye-polymer interaction. The values of $\mathrm{k}_{\mathrm{nr}} / \mathrm{k}_{\mathrm{r}}$ for the dye are calculated in the PEI media. The changing photophysical parameter values of the RB are due to the local micro viscosity and polarity variations as well as the probe surrounding interactions in this medium and dye interactions. Changing and limiting the photophysical properties of the dye with the concentration of the polymer will be beneficial tools used in optical studies.

\section{ACKNOWLEDGEMENT}

The general support by Atatürk University is gratefully acknowledged.

\section{REFERENCES}

1. Bayraktutan $\mathrm{T}$, Onganer $\mathrm{Y}$, Meral $\mathrm{K}$. Polyelectrolyte-induced $\mathrm{H}$-aggregation of Merocyanine 540 and its application in metal ions detection as a colorimetric sensor. Sens. Actuators, B . 2016;226:52-61.

2. Takagishi T, Yoshikawa K, Hamano H, Kuroki $\mathrm{N}$, Kozuka $\mathrm{H}$. Specific Interaction Between Polyethylenimine And Azo Dyes Carrying Hydroxyl-Groups. J. Polym. Sci., Part A: Polym. Chem. . 1985;23(1):37-47.

3. Kunath $K$, von Harpe A, Fischer D, Peterson $H$, Bickel U, Voigt $K$, et al. Low-molecular-weight polyethylenimine as a non-viral vector for DNA delivery: comparison of physicochemical properties, transfection efficiency and in vivo distribution with high-molecular-weight polyethylenimine. J. Controlled Release. 2003;89(1):113-25.

4. Bisset $W$, Jacobs $H$, Koshti N, Stark P, Gopalan A. Synthesis and metal ion complexation properties of a novel polyethyleneimine $\mathrm{N}$ methylhydroxamic acid water soluble polymer. React. Funct. Polym. 2003;55(2):109-19.

5. Kobayashi S, Hiroishi K, Tokunoh M, Saegusa T. Chelating Properties Of Linear And Branched Poly(Ethylenimines).

1987;20(7):1496-500.

Macromol.

6. Amara $M$, Kerdjoudj $H$. Modification of the cation exchange resin properties by impregnation in polyethyleneimine solutions - Application to the separation of metallic ions. Talanta. 2003;60(5):991-1001.

7. Neckers DC. Rose-Bengal. J. Photochem. Photobiol., A 1989;47(1):1-29.

8. Chang CC, Yang YT, Yang JC, Wu HD, Tsai T. Absorption and emission spectral shifts of rose bengal associated with DMPC liposomes. Dyes Pigm. 2008;79(2):170-
9. Bayraktutan T, Onganer Y. Biophysical influence of coumarin 35 on bovine serum albumin: Spectroscopic study. Spectrochim. Acta, Part A . 2017;171:90-6.

10. Toprak M, Arik M. An investigation of energy transfer between coumarin 35 and xanthene derivatives in liquid medium. Turk. J. Chem. 2010;34(2):285-93.

11. Atahan A, Orhan E. Photophysics, pH Sensing and Hydrolysis Study of a Novel 1,8Naphthalimide Derivative. J. Turk. Chem. Soc., Sect. A: Chem. 2018;5(2):775-784.

12. Kubin RF, Fletcher AN. Fluorescence Quantum Yields Of Some Rhodamine Dyes. J. Lumin. $1982 ; 27(4): 455-62$.

13. Vaitekonis S, Trinkunas G, Valkunas L. Red chlorophylls in the exciton model of photosystem I. Photosynth. Res. 2005;86(1-2):185-201.

14. Gungor O, Durmus M, Ahsen V. Investigation of photochemical and photophysical properties of novel silicon(IV) phthalocyanines and their muoxo dimers. Turk. J. Chem. 2017;41(6):803-8.

15. Moczek L, Nowakowska M. Novel watersoluble photosensitizers from chitosan. Biomacromol. 2007;8(2):433-8.

16. Maruthamuthu $M$, Reddy JV. Binding Of Fluoride Onto Poly(N-Vinyl-2-Pyrrolidone). J. Polym. Sci., Part C: -Polym. Lett. 1984;22(10):569-73.

17. Hong YN, Lam JWY, Tang BZ. Aggregationinduced emission. Chem. Soc. Rev. 2011;40(11):5361-88.

18. Güzel E. Preparation and investigation of aggregation, fluorescence and singlet oxygen generation properties of gallium and metal-free phthalocyanines. J. Turk. Chem. Soc., Sect. A: Chem. 2019;5(3):1051-60.

19. Wu WC, Chen CY, Tian YQ, Jang SH, Hong YN, Liu $Y$, et al. Enhancement of Aggregation-Induced Emission in Dye-Encapsulating Polymeric Micelles for Bioimaging. Adv. Funct. Mater. $2010 ; 20(9): 1413-23$.

20. Chen JW, Law CCW, Lam JWY, Dong YP, Lo SMF, Williams ID, et al. Synthesis, light emission, nanoaggregation, and restricted intramolecular rotation of 1,1-substituted 2,3,4,5tetraphenylsiloles. Chem. Mater. 2003;15(7):1535-46.

21. Block MAB, Hecht S. Poly(propylene oxide)poly(phenylene ethynylene) block and graft copolymers. Macromol. 2008;41(9):3219-27. 
22. Wehry EL. Principles Of Fluorescence Spectroscopy - Lakowicz,JR. Am. Sci. $1984 ; 72(4): 395-6$.

23. Lepecq JB. Citation Classic - A Fluorescent Complex Between Ethidium-Bromide And NucleicAcids - Physical-Chemical Characterization. Current Contents/Life Sci. 1984(35):16-20.

24. Datta A, Mandal D, Pal SK, Bhattacharyya K. Intramolecular charge transfer processes in confined systems. Nile red in reverse micelles. J. Phys. Chem. B. 1997;101(49):10221-5.

25. Ozcelik S, Atay NZ. Optical transition rates of a meso-substituted thiacarbocyanine in methanol-in-oil reverse micelles (vol 113, pg 1,
2005). J. Lumin. $2005 ; 114(3-4): 314-8$.

26. Thomas SW, Joly GD, Swager TM. Chemical sensors based on amplifying fluorescent conjugated polymers. Chem. Rev. 2007;107(4):1339-86.

27. Gur B, Meral K. The effect of poly(vinyl alcohol) on the photophysical properties of pyronin dyes in aqueous solution: A spectroscopic study. Spectrochim. Acta, Part A. 2013;101:30613.

28. Bayraktutan T, Meral K, Onganer $\mathrm{Y}$. Photophysical properties of pyronin dyes in reverse micelles of AOT. J. Lumin. 2014;145:9259. 